\title{
XDR Surveillance in a Tertiary Care Hospital: A Periodic Observational Study
}

\author{
Jose John', Juby O. Chacko', Johnson V. Babu',** \\ ${ }^{1}$ Department of Clinical Pharmacy, Caritas Hospital, Thellakom, Kerala, INDIA. \\ ${ }^{2}$ Department of Pharmacy Practice, Nirmala College of Pharmacy, Muvattupuzha, Kerala, INDIA.
}

\begin{abstract}
Background: The study focused on patients with XDR organisms and the risk factors to analyse the increase in trend of XDR colonised/infected patients from the community and hospital setting. Methodology: The study employed a periodic observational study conducted in a tertiary care hospital. The periodic selection of duration was based on the changes in antimicrobial resistance pattern identified previously in the hospital. The periods chosen was November-December 2018, May-June 2019, NovemberDecember 2019, May-June 2020 and November- December 2020. The study did not involve any sampling or experiment during the data procurement process even if it was a prospective study. The whole required details obtained from the patient medical record and microbiology laboratory. Results: The percentage of XDR isolate obtained were $5-6 \%$ from the total culture specimen. Klebsiella was the most identified organism in our study $(70 \%)$. Initially colonisation was highest, when moving forward number of infected patients were rose. Major risk factors identified were prolonged antibiotic exposure $(>50 \%)$, previous hospitalisation $(>40 \%)$, catheter $(70 \%)$, age (mean age- 58.2 years). The average hospitalisation duration was increased 3 times when compared normal hospitalisation. Conclusion: Although a few prospective studies have examined the relationship between the details of antibiotic exposure and resistance development, differentiating particular factors related to prior antimicrobial exposure and the development of resistance has been challenging.
\end{abstract}

Key words: XDR, Resistance, Antibiotics, Infection, Colonisation.

\section{INTRODUCTION/ BACKGROUND}

Antibiotic resistance is a worldwide health warning, and both national and international efforts have underscored the need to minimize antimicrobial resistance. In 2014, the Centers for Disease Control and Prevention (CDC) called for hospitals to have antibiotic stewardship programs, defined as coordinated evidence-based efforts to optimize antibiotic use. ${ }^{1}$

In 2016, at the $71^{\text {st }}$ United Nations General Assembly, world leaders pledged to combat the spread of resistance, and since 2017, hospitals have been mandated by the Joint Commission to implement antibiotic stewardship programs. However, the implementation of stewardship programs has mainly focused on clinicians and pharmacists.

The study findings of Giancarlo et al. 2018 underline an increased circulation of multidrug-resistant organism within the hospital, and the acquisition of antibiotic resistance mechanism. ${ }^{2}$

Management and controlling measure remain difficult for a clinician/ epidemiologist depending on the extent of drug resistance to antibiotics, both empirically and routinely after ascertaining the antibiograms of isolates. ${ }^{3}$ Antibiotic use is increasing worldwide and has been implicated in the dramatic rise in emergence of antibiotic-resistant organisms which is a
DOI: 10.5530/ijopp.14.4.56

Address for correspondence: Dr. Johnson V. Babu, Pharm.D,

Nirmala College of Pharmacy, Muvattupuzha-686661, Kerala, INDIA.

Phone no: +91 8281325336 Email id: johnsonbabuz@ gmail.com

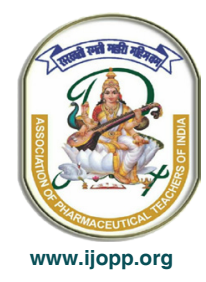


major public health concern, particularly in hospitals and other health care settings. Antibiotic-resistant organisms appear to be biologically and genetically fit and have potential to cause serious, life-threatening infections that are difficult to control because treatment options are narrow. 4.5

There was a slight increase in asymptomatic patients with extensive drug resistance (XDR) isolates in the culture sensitivity reports which led to numerous inappropriate therapies. Chances of contamination was important for a wrong result which may lead to inappropriate antibiotic therapy. Hence to overcome the problem, a prospective surveillance of XDR patients was done. A rise in the number of XDR isolates reflected the number of high-end antibiotics consumed.

Increase in the prevalence of drug-resistance is occurring at a time when new anti-infective agent's discovery is slowing down dramatically. We face a growing number of potentially untreatable infections not-too-distant future. ${ }^{5}$

This study focused on patients with XDR organisms and the type of treatment and other details to analyse the increase in trend of XDR colonised patients from the community and hospital setting.

\section{METHODOLOGY}

The study employed a periodic observational study conducted in a tertiary care hospital. The periodic selection of duration was based on the changes in antimicrobial resistance pattern identified previously in the hospital. The study period selected was NovemberDecember 2018, May-June 2019, November- December 2019, May-June 2020 and November- December 2020. To identify the significant changes in the pattern of resistance periodic study was choose. The study did not involve any sampling or experiment during the data procurement process even if it was a prospective study. The whole required details obtained from the patient medical record and microbiology laboratory. The data were only accessible to researchers and ensured that third party didn't have access to the raw information collected. In-patients with XDR isolates confirmed with culture were included in the study. We excluded outpatients due to difficulty in data procurement. The data collection form used for data procurement pretested with a short-term pilot study and ensured its validity. The questions are framed in such a way that it accounts for demographics, isolate details, treatment, history of antibiotic exposure, hospital stay, specimen details etc.
All the analysis and calculation were done using excel. The data obtained during the study were statistically analyzed by central frequencies and percentage.

\section{RESULTS}

The basic parameters including demographics, total sample, previous hospitalisation was tabulated in Table 1. A total of 27840 patients were admitted to the hospital during this study. About 209 XDR isolate obtained from 11444 specimens. Patients with $>60$ years and male gender were identified with the greatest number of $\mathrm{XDR}$ in all period. Most of the patients were previously admitted to the hospital within the past 90 days.

Figure 1 illustrating the antibiotic exposure in the past 90 days of patients enrolled in the study during the various study period, which reported that the number of patients exposed with antibiotic is higher than those who not exposed in all period except May-June 2020. All other period had more than 50\% exposure. The usage was reduced in May-June 2020 due to the COVID pandemic.

Antibiotics used during the previous admission showed that cefoperazone/sulbactam, meropenem, levofloxacin, nitrofurantoin were mostly used for the treatment. Restricted antibiotics also were prescribed along with other ones. During May-June 2019 the usage of antibiotics was less when compared to other periods (Figure 2).

The major source of infection found to be urinary tract infection during the first, second and fourth phase of the study. During the third and fifth skin/soft tissue infection found to be high as the source of infection. The XDR isolates identified from other infection source was less compared to urinary tract infection and skin/ soft tissue infection. The previous XDR isolate with the same organism identified was $27.41 \%$ during the first phase and follows as $16.12 \%, 31.11 \%, 18.75 \%, 33.33 \%$ in other phases. Previous XDR isolates with different organism were $4.8 \%$ in first phase, $3.22 \%$ in the second phase, $2.22 \%$ in the third phase, $9.37 \%$ in the fourth phase, $7.69 \%$ in the fifth phase. Patients with history of MDR/XDR isolates with the same organism was found to be very high in the last phase with $35.9 \%$ and $19.35 \%$, $16.12 \%, 6.67 \%, 9.37 \%$ as follows from the first phase to the fourth phase. More than $75 \%$ of patients had urinary catheter except the first phase with 67.2\% (Table 2).

Antibiotics used during current admission of infection showed that meropenem was the most used antibiotic. 
Table 1: Basic Parameters and demographics.

\begin{tabular}{|c|c|c|c|c|c|c|}
\hline & & Nov-Dec 2018 & May-Jun 2019 & Nov-Dec 2019 & May-Jun 2020 & Nov-Dec 2020 \\
\hline \multicolumn{2}{|c|}{ Total no of patients admitted } & 5898 & 6200 & 6185 & 4615 & 4942 \\
\hline \multicolumn{2}{|c|}{ Total of XDR patients } & $58(0.98 \%)$ & $22(0.35 \%)$ & $32(0.52 \%)$ & $25(0.54 \%)$ & $29(0.59 \%)$ \\
\hline \multicolumn{2}{|c|}{ Total samples tested } & 2496 & 2625 & 2409 & 1887 & 2027 \\
\hline \multicolumn{2}{|c|}{ Total isolates obtained } & $905(36.2 \%)$ & $956(36.4 \%)$ & $726(30.1 \%)$ & $589(31.2 \%)$ & $628(31 \%)$ \\
\hline \multicolumn{2}{|c|}{ Total XDR isolates } & $62(6.8 \%)$ & $31(3.24 \%)$ & $45(6.2 \%)$ & $32(5.4 \%)$ & $39(6.2 \%)$ \\
\hline & $\leq 18$ years & $1(1.72 \%)$ & - & - & - & $2(6.9 \%)$ \\
\hline \multirow[t]{2}{*}{ Age } & $19-60$ years & $18(31.03 \%)$ & $8(36.4 \%)$ & $13(40.6 \%)$ & $11(44 \%)$ & $13(44.8 \%)$ \\
\hline & $>60$ years & $39(67.2 \%)$ & $14(63.6 \%)$ & $19(59.4 \%)$ & $14(56 \%)$ & $14(48.27 \%)$ \\
\hline \multirow{3}{*}{ Gender } & Male & $34(58.6 \%)$ & $15(68.2 \%)$ & $20(62.5 \%)$ & $16(64 \%)$ & $21(72.4 \%)$ \\
\hline & Female & $24(41.37 \%)$ & $7(31.8 \%)$ & $12(37.5 \%)$ & $9(28.12 \%)$ & $9(31.03 \%)$ \\
\hline & Zero & $8(13.8 \%)$ & $4(18.18 \%)$ & $13(40.62 \%)$ & $10(40 \%)$ & $8(27.6 \%)$ \\
\hline \multirow{4}{*}{$\begin{array}{c}\text { No. of previous } \\
\text { hospitalisations } \\
\text { past } 90 \text { days }\end{array}$} & One & $29(50 \%)$ & $12(54.54 \%)$ & $12(37.5 \%)$ & $11(44 \%)$ & $9(31.03 \%)$ \\
\hline & Two & $16(27.59 \%)$ & $3(13.63 \%)$ & $7(27.87 \%)$ & $2(8 \%)$ & $9(31.03 \%)$ \\
\hline & Three & $4(6.9 \%)$ & $4(18.18 \%)$ & - & $2(8 \%)$ & $3(10.3 \%)$ \\
\hline & four & $1(1.72 \%)$ & - & - & - & - \\
\hline
\end{tabular}

Abbreviations: Nov-November, Dec- December, Jun- June, IP- inpatients, OP-outpatients

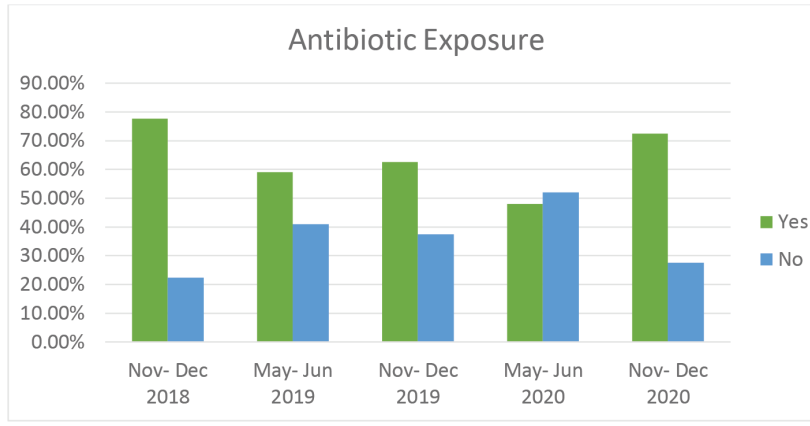

Figure 1: Antibiotic exposure in past 90 days.

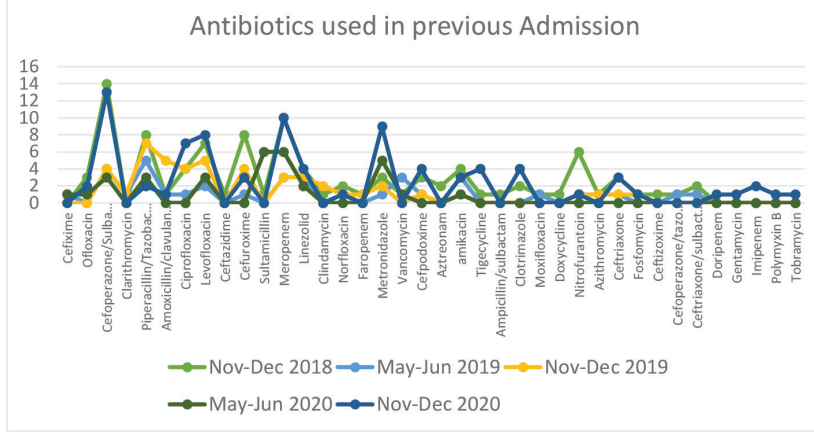

Figure 2: Antibiotics used in previous admission.

Apart from this cefoperazone/sulbactam, Levofloxacin, ofloxacin, metronidazole, and polymyxin $\mathrm{B}$ are the others. November December 2018 reported with the highest amount of antibiotics prescribed. (Figure 3)

The identified XDR organisms were klebsiella, pseudomonas, Acinetobacter, E. coli, Enterobacter,
Citrobacter. Among these mostly isolated organisms was Klebsiella in all phases with $62.9 \%, 87.1 \%$, $62.22 \%, 62.5 \%, 76.62 \%$. And the least identified organism was Citrobacter with $3.22 \%$ in May- June 2019. Contamination/ colonisation defined as the type of growth in which the patient had no symptoms unlike what is seen in infected patients. The majority of identified isolates were contaminated/ colonised initially and gradually showed an increase in infection towards the end phase. 66 patients were found to had community-acquired infection altogether and 103 patients had prolonged exposure to antibiotics as a risk factor for XDR. Hospital-acquired/ health careassociated infection was the most identified route of infection. The therapy was found to be appropriate in all case except a few (11 cases) in all phases. Patient with XDR isolates stayed in the hospital for 11-15 days, however the hospital average of hospital stay is approximately 4 days. (Table 3 )

The major antibiotics given for XDR isolated patient were meropenem, tigecycline, polymyxin $\mathrm{B}$, Colistin, Fosfomycin, cefoperazone/sulbactam. (Figure 4)

\section{DISCUSSION}

Patients who are infected with XDR often have an increased risk of prolonged illness and mortality. The cost of care for their patients can be more than double when compared to those without. ${ }^{6}$ In our study, a total of less than $1 \%$ of patients had XDR in all periods and it was less than $0.6 \%$ in all other periods except 
Table 2: Type, sample, source and history of infection.

\begin{tabular}{|c|c|c|c|c|c|c|}
\hline & & Nov-Dec 2018 & $\begin{array}{l}\text { May-Jun } \\
2019\end{array}$ & $\begin{array}{l}\text { Nov-Dec } \\
2019\end{array}$ & May-Jun 2020 & $\begin{array}{l}\text { Nov-Dec } \\
2020\end{array}$ \\
\hline \multirow{5}{*}{ 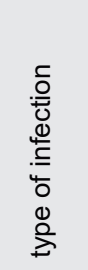 } & Urinary tract infection & $33(53.2 \%)$ & $14(45.16 \%)$ & $14(31.11 \%)$ & $11(34.37 \%)$ & $9(23.07 \%)$ \\
\hline & Skin and soft tissue infection & $10(16.13 \%)$ & $2(6.45 \%)$ & $22(48.89 \%)$ & $6(18.75 \%)$ & $22(56.41 \%)$ \\
\hline & Respiratory tract infection & $9(14.52 \%)$ & $9(29.03 \%)$ & $2(4.44 \%)$ & $10(31.25 \%)$ & $3(7.69 \%)$ \\
\hline & Urosepsis & $4(6.45 \%)$ & $1(3.22 \%)$ & $1(2.22 \%)$ & - & - \\
\hline & Blood stream infection & $3(4.8 \%)$ & $5(16.12 \%)$ & $6(13.33 \%)$ & $1(3.12 \%)$ & $5(12.82 \%)$ \\
\hline \multirow{6}{*}{ 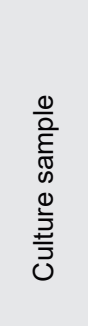 } & Others* & $3(4.8 \%)$ & - & - & $4(12.5 \%)$ & - \\
\hline & Urine & $34(54.83 \%)$ & $14(45.16 \%)$ & $14(31.11 \%)$ & $11(34.37 \%)$ & $9(23.07 \%)$ \\
\hline & Pus & $12(19.35 \%)$ & $2(6.45 \%)$ & $22(48.89 \%)$ & $9(28.12 \%)$ & $19(48.71 \%)$ \\
\hline & Blood & $4(6.45 \%)$ & $6(19.35 \%)$ & $7(15.55 \%)$ & $1(3.12 \%)$ & $5(12.82 \%)$ \\
\hline & Sputum & $9(14.52 \%)$ & $9(29.03 \%)$ & $2(4.444 \%)$ & $3(9.37 \%)$ & $3(7.69 \%)$ \\
\hline & Others ${ }^{* *}$ & $3(4.8 \%)$ & - & - & $8(25 \%)$ & $3(7.69 \%)$ \\
\hline \multirow{2}{*}{$\frac{\alpha}{2} \frac{i}{0}$} & $\begin{array}{c}\text { Previous XDR isolate with same } \\
\text { organism }\end{array}$ & $17(27.41 \%)$ & $5(16.12 \%)$ & $14(31.11 \%)$ & $6(18.75 \%)$ & $13(33.33 \%)$ \\
\hline & $\begin{array}{c}\text { Previous XDR isolate with different } \\
\text { organism }\end{array}$ & $3(4.8 \%)$ & $1(3.22 \%)$ & $1(2.22 \%)$ & $3(9.37 \%)$ & $3(7.69 \%)$ \\
\hline \multirow{2}{*}{ 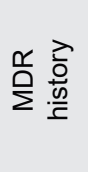 } & $\begin{array}{c}\text { Previous MDR isolate with same } \\
\text { organism }\end{array}$ & $12(19.35 \%)$ & $5(16.12 \%)$ & $3(6.67 \%)$ & $3(9.37 \%)$ & $14(35.9 \%)$ \\
\hline & $\begin{array}{c}\text { Previous MDR isolate with different } \\
\text { organism }\end{array}$ & $6(9.68 \%)$ & - & $3(6.67 \%)$ & - & $3(7.69 \%)$ \\
\hline \multirow{2}{*}{ 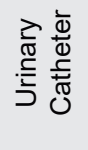 } & Yes & $39(67.2 \%)$ & $21(95.45 \%)$ & $26(81.25 \%)$ & $19(76 \%)$ & $24(82.76 \%)$ \\
\hline & No & $19(32.76 \%)$ & $1(4.55 \%)$ & $6(18.75 \%)$ & $6(24 \%)$ & $5(17.24 \%)$ \\
\hline
\end{tabular}

Other*-CNS infection, otitis media, intraabdominal infection

Others**-Broncho-alveolar lavage, body fluid, ear swab, CSF

Abbreviations: XDR-extensive drug resistance, MDR-multi drug resistance.

Antibiotics used in Current admission

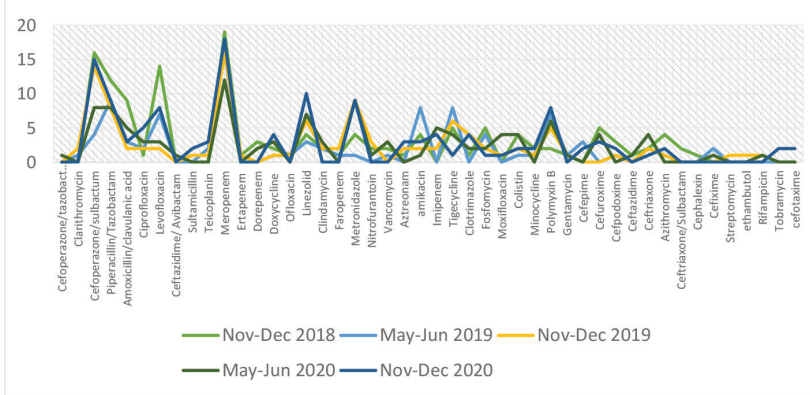

Figure 3: Antibiotics used in Current admission.

November- December 2018 because of contamination/ colonisation. Silpi et al. 2016 reported that in central India there were about $1.6 \%$ of patients with XDR. Also, the total isolates we obtained were half percentage of the Silpi et al. 2016 (12.1\%). the main reasons behind the reduction were the proper infection control practice, antibiotic usage, personal hygiene, well-established health care system, etc. ${ }^{7}$
Patients with > 60 years identified more with XDR isolates in all periods when compared with other age groups. The mean age of patients in our study was 58.2 years but Bahman et al. 2020 had 49.8 years. The chance of comorbidity is high among the aged population which lead to increased hospitalisation. ${ }^{8}$

Both our study and Bahman et al. 2020 reported that males have more XDR when compared to females. Because many males have social habits like smoking, alcoholism etc which lead to reduced immunity and other comorbidities leading to hospitalisation causing resistance. ${ }^{8}$ In males the cellulitis infection is high and in female the urinary tract infection is high. The Center for Disease Dynamics, Economics and Policy (CDDEP) reported that drug-resistant infections are higher among in-patients with previous hospitalisation. ${ }^{9}$ In our study, more than $60 \%$ had one-time hospitalisation. The ICU stay has also an impact on the occurrence of resistance. 


\section{Table 3: Isolate details and Therapy}

\begin{tabular}{|c|c|c|c|c|c|c|}
\hline & & Nov-Dec 2018 & May-Jun 2019 & Nov-Dec 2019 & May-Jun 2020 & Nov-Dec 2020 \\
\hline \multirow{6}{*}{$\begin{array}{l}\text { Organism } \\
\text { Isolated }\end{array}$} & Klebsiella & $39(62.9 \%)$ & $27(87.1 \%)$ & $28(62.22 \%)$ & $20(62.5 \%)$ & $30(76.62 \%)$ \\
\hline & Pseudomonas & $18(29.3 \%)$ & $1(3.22 \%)$ & $5(11.11 \%)$ & $1(3.12 \%)$ & $5(12.82 \%)$ \\
\hline & Acinetobacter & $4(6.45 \%)$ & - & $10(22.22 \%)$ & $4(12.5 \%)$ & $2(5.13 \%)$ \\
\hline & E. Coli & $1(1.61 \%)$ & - & $2(4.44 \%)$ & $5(15.62 \%)$ & - \\
\hline & Enterobacter & - & $2(6.45 \%)$ & - & $2(6.25 \%)$ & $2(5.13 \%)$ \\
\hline & Citrobacter & - & $1(3.22 \%)$ & - & - & - \\
\hline \multirow{3}{*}{$\begin{array}{l}\text { Type of } \\
\text { growth }\end{array}$} & $\begin{array}{l}\text { Contamination/ } \\
\text { Colonisation }\end{array}$ & $47(75.8 \%)$ & $15(48.38 \%)$ & $20(44.44 \%)$ & $16(50 \%)$ & $14(35.9 \%)$ \\
\hline & Infection & $15(24.19 \%)$ & $16(51.61 \%)$ & $25(55.56 \%)$ & $16(50 \%)$ & $25(64.1 \%)$ \\
\hline & $\begin{array}{l}\text { Community } \\
\text { acquired }\end{array}$ & $18(29 \%)$ & $7(22.58 \%)$ & $17(37.78 \%)$ & $14(43.75 \%)$ & $10(25.64 \%)$ \\
\hline \multirow[t]{2}{*}{$\begin{array}{l}\text { Route of } \\
\text { infection }\end{array}$} & $\begin{array}{l}\text { Hospital acquired/ } \\
\text { health care } \\
\text { associated }\end{array}$ & $34(54.84 \%)$ & $18(58.06 \%)$ & $23(51.11 \%)$ & $14(43.75 \%)$ & $19(48.71 \%)$ \\
\hline & $\begin{array}{l}\text { Prolonged } \\
\text { exposure to } \\
\text { antibiotics }\end{array}$ & $10(16.13 \%)$ & $6(19.35 \%)$ & $5(11.11 \%)$ & $4(12.5 \%)$ & $10(25.64 \%)$ \\
\hline \multirow{2}{*}{ Therapy } & Inappropriate & $4(6.9 \%)$ & $1(4.54)$ & $3(9.37 \%)$ & $3(12 \%)$ & - \\
\hline & Appropriate & $54(93.1 \%)$ & $19(95.44 \%)$ & $29(90.62 \%)$ & $22(88 \%)$ & $29(100 \%)$ \\
\hline \multirow{6}{*}{$\begin{array}{l}\text { Duration of } \\
\text { hospital stay }\end{array}$} & $0-5$ days & $9(15.51 \%)$ & $2(9.1 \%)$ & $6(18.75 \%)$ & $5(20 \%)$ & $6(20.69 \%)$ \\
\hline & $6-10$ days & $12(20.69 \%)$ & $4(18.18 \%)$ & $6(18.75 \%)$ & $5(20 \%)$ & $3(10.34 \%)$ \\
\hline & $11-15$ days & $18(31.03 \%)$ & $3(13.64 \%)$ & $4(12.5 \%)$ & $6(24 \%)$ & $7(24.13 \%)$ \\
\hline & $16-20$ days & $6(10.34 \%)$ & $5(22.72 \%)$ & $4(12.5 \%)$ & $3(12 \%)$ & $4(13.8 \%)$ \\
\hline & $>20$ days & $8(13.79 \%)$ & $4(18.18 \%)$ & $6(18.75 \%)$ & $1(4 \%)$ & $2(6.9 \%)$ \\
\hline & $>1$ month & $5(8.62 \%)$ & $4(18.18 \%)$ & $6(18.75 \%)$ & $5(20 \%)$ & $7(24.13 \%)$ \\
\hline
\end{tabular}

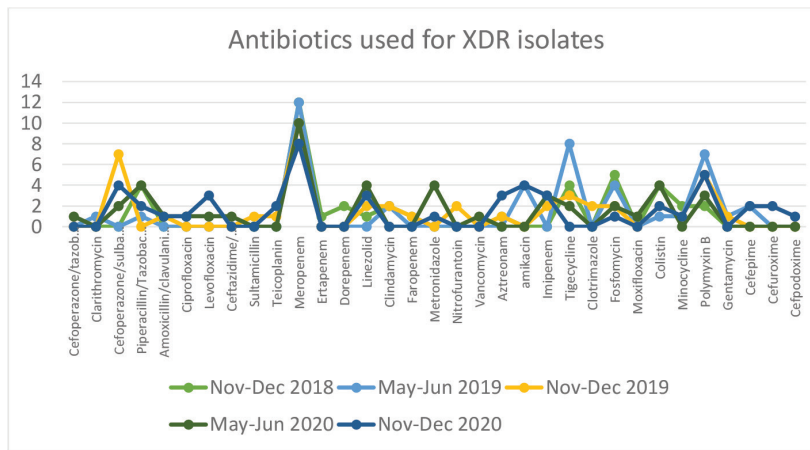

Figure 4: Antibiotics used for XDR isolates.

Although a few prospective studies have examined the relationship between the details of antibiotic exposure and resistance development, differentiating particular factors related to prior antimicrobial exposure and the development of resistance has been challenging. ${ }^{10,11}$

In our study the rate of previous antibiotic exposure is between $40 \%$ to $90 \%$ which has an impact on antibiotic resistance.

The majority of antibiotic given previously was board spectrum because many of the patients had MDR or
XDR previously. Therefore, the need for board spectrum antibiotics as empirical therapy was essential.

In our study most organisms were isolated from urine and pus specimen, therefore UTI and SSTI were found to be the source of infection for most. The study conducted by Ravichandran et al. 2015 also inferred with the same result that XDR isolates found most in urine followed by pus. The presence of a catheter is an important reason that leads to an increased rate of XDR organisms in urine. ${ }^{12}$ Joyce wang et al. 2017 supports this result through their study. ${ }^{13}$ As per their study presence of a catheter may lead to bacterial colonisation which will eventually lead to infection.

A P Margiorakos et al. 2012 and Joyce wang et al. 2017 says antibiotic use is one of the major risk factors for MDR, XDR colonisation which in turn lead to crossresistance. ${ }^{13,14}$ From our study it is clear that patients with previous MDR/XDR colonisation with the same or different organisms have a higher chance of developing colonisation/infection with XDR organisms

Empirically for the current admission board, spectrum antibiotic was chosen because many of them had the 
previous history and also, they were prescribed with board spectrum previously.

In all five phases of study $>60 \%$ of isolated organism was Klebsiella XDR organism, followed by pseudomonas $(>14 \%)$ and Acinetobacter. The study conducted by Silpi et al. 2016 observed that pseudomonas was the most identified organism followed by klebsiella. Compared to Silpi et al. 2016 in our study klebsiella was higher in number. ${ }^{7}$

Previous studies said that infection was greater than contamination/ colonisation but in our study in the initial period number of patients with contamination/ colonisation was high. ${ }^{7-11}$ Gradually the pattern was changed to a hike in infection type of growth. The major reason behind the increased level of contamination/ colonisation was due to sample collection error and it was rectified by taking necessary steps under the infection control department.

The reason for the declined percentage of communityacquired infection towards the end can be due to the COVID pandemic. The interpersonal involvement, improved personal hygiene, less exposure to hospital/ antibiotics, usage of mask and sanitiser were helped in the reduction of community-acquired infection. Most of the patient had 11-16 days of hospital stay. This includes both discharged and expired patients. Based on the identification of the organism appropriate therapy was given.

Some of our limitations were lack of proper guidelines and articles regarding to epidemiological data of XDR. We had done a periodical study rather than a continuous study.

\section{CONCLUSION}

Infections with XDR organisms are increasing day by day with high treatment cost and mortality rate. In our study, the rate was between 5-7\%. The rate of resistance of klebsiella is increasing. Initially, it was colonisation and later infection found to be most predominant. Major reasons for the XDR colonisation/infection are previous antibiotic exposure, previous hospitalisation, presence of a urinary catheter, history of MDR/XDR colonisation/infection etc. UTI and SSTI were the most common sources of infection identified. Communityacquired XDR infections/colonisation are presently increasing in patients without any health care-associated risk factors.

\section{ACKNOWLEDGEMENT}

Nil

\section{CONFLICT OF INTEREST}

The authors declare no Conflict of interest.

\section{ABBREVIATIONS}

CDC: Centers for Disease Control and Prevention; CDDEP: The Center for Disease Dynamics, Economics and Policy; ICU: Intensive Care Unit; MDR: Multi-drug Resistance; SSTI: Skin and Soft tissue infection; UTI: Urinary tract infection; XDR: extensive drug resistance.

\section{SUMMARY}

A periodic observational study conducted in a tertiary care hospital antimicrobial resistance pattern. 5-6\% was the XDR organism identified from the total culture isolation. Among this Klebsiella found to be the highest in percentage with $70 \%$. Major risk factors identified were prolonged antibiotic exposure, previous hospitalisation, catheter. The usage of antibiotics and its effect also discussed in this study. The study revealed that average hospitalisation will increase thrice when XDR infection hit the patient. XDR infections are increasing day by day which lead to increased economic burden and mortality.

\section{REFERENCES}

1. Carter EJ, Greendyke WG, Furuya EY, Srinivasan A, Shelley AN, Bothra A, et al. Exploring the nurses' role in antibiotic stewardship: A multisite qualitative study of nurses and infection preventionists. Am J Infect Control. 2018;46(5):492-7. doi: 10.1016/j.ajic.2017.12.016, PMID 29395509. PMCID PMC6495548.

2. Ripabelli G, Tamburro M, Guerrizio G, Fanelli I, Flocco R, Scutellà M, et al. Tracking multidrug-resistant Klebsiella pneumoniae from an Italian Hospital: molecular epidemiology and surveillance by PFGE, RAPD and PCR-based resistance genes prevalence. Curr Microbiol. 2018;75(8):977-87. doi: 10.1007/ s00284-018-1475-3, PMID 29523910.

3. Rath S, Padhy RN. Prevalence of two multidrug-resistant Klebsiella species in an Indian teaching hospital and adjoining community. J Infect Public Health. 2014;7(6):496-507. doi: 10.1016/j.jiph.2014.05.002, PMID 24996691.

4. Bharadwaj R, Robinson ML, Balasubramanian U, Kulkarni V, Kagal A, Raichur P, et al. Drug-resistant Enterobacteriaceae colonization is associated with healthcare utilization and antimicrobial use among inpatients in Pune, India. BMC Infect Dis. 2018;18(1):504. doi: 10.1186/s12879-018-3390-4, PMID 30286741.

5. Mulvey MR, Simor AE. Antimicrobial resistance in hospitals: how concerned should we be? CMAJ. 2009;180(4):408-15. doi: 10.1503/cmaj.080239, PMID 19221354. PMCID PMC2638041.

6. Lange C, Abubakar I, Alffenaar JW, Bothamley G, Caminero JA, Carvalho AC, et al. Management of patients with multidrug-resistant/ extensively drug-resistant tuberculosis in Europe: a TBNET consensus statement. Eur Respir J. 2014;44(1):23-63. doi: 10.1183/09031936.00188313, PMID 24659544. 
7. Basak S, Singh P, Rajurkar M. Multidrug resistant and extensively drug resistant bacteria: A study. J Pathog. 2016;2016:4065603. doi: 10.1155/2016/4065603, PMID 26942013, PMCID PMC4749793.

8. Mirzaei B, Bazgir ZN, Goli HR, Iranpour F, Mohammadi F, Babaei R. Prevalence of multi-drug resistant (MDR) and extensively drug-resistant (XDR) phenotypes of Pseudomonas aeruginosa and Acinetobacter baumannii isolated in clinical samples from Northeast of Iran. BMC Res Notes. 2020;13(1):380. doi: 10.1186/ s13104-020-05224-w, PMID 32778154.

9. Drug resistant infections associated with higher in-hospital mortality rates in India. [ebook] Center for Disease Dynamics, Economics and Policy (CDDEP). Vol. 2.

10. Costelloe C, Metcalfe C, Lovering A, Mant D, Hay AD. Effect of antibiotic prescribing in primary care on antimicrobial resistance in individual patients: systematic review and meta-analysis. BMJ. 2010;340:c2096. doi: 10.1136/bmj. c2096, PMID 20483949
11. Kuster SP, Rudnick W, Shigayeva A, Green K, Baqi M, Gold WL, et al. Previous antibiotic exposure and antimicrobial resistance in invasive pneumococcal disease: results from prospective surveillance. Clin Infect Dis. 2014;59(7):94452. doi: 10.1093/cid/ciu497, PMID 24973312.

12. Ravichandran $M$, Munisamy $P$, Chandrasekar V. Demographical study of extensive drug-resistant Gram-negative bacteria with precise attention on XDR uropathogen E. coli. Int J Curr Microbiol Appl Sci. 2015;4(10):794-806.

13. Wang J, Foxman B, Mody L, Snitkin ES. Network of microbial and antibiotic interactions drive colonization and infection with multidrug-resistant organisms. Proc Natl Acad Sci U S A. 2017;114(39):10467-72. doi: 10.1073/ pnas.1710235114, PMID 28900004.

14. Magiorakos AP, Srinivasan A, Carey RB, Carmeli Y, Falagas ME, Giske CG, et al. Multidrug-resistant, extensively drug-resistant and pandrug-resistant bacteria: an international expert proposal for interim standard definitions for acquired resistance. Clin Microbiol Infect. 2012;18(3):268-81. doi: 10.1111/j.14690691.2011.03570.x, PMID 21793988. 